\title{
Influence of the insemination method on the outcomes of elective blastocyst culture
}

\author{
Caizhu Wang*, Guixue Feng*, Bo Zhang, Jinhui Shu, Hong Zhou, Xianyou Gan, Ruoyun Lin \\ Center of Reproductive Medicine, Guangxi Maternal and Child Health Hospital, Nanning, China
}

Objective: The aim of this study was to explore the effects of the insemination method on the outcomes of elective blastocyst culture.

Methods: We retrospectively analyzed the outcomes of elective blastocyst culture performed between January 2011 and December 2014.

Results: There were 2,003 cycles of conventional in vitro fertilization (IVF) and 336 cycles of intracytoplasmic sperm injection (ICSI), including 25,652 and 4,164 embryos that underwent sequential blastocyst culture, respectively. No significant differences were found in the female patients' age, basal follicle-stimulating hormone level, basal luteinizing hormone level, body mass index, number of oocytes, maturity rate, fertilization rate, or good-quality embryo rate. However, the blastocyst formation rate and embryo utilization rate were significantly higher in the conventional IVF group than in the ICSI group $(54.70 \%$ vs. $50.94 \%$ and $51.09 \%$ vs. $47.65 \%$, respectively, $p<0.05)$. The implantation/pregnancy rate (IVF, 50.93\%; ICSI, 55.10\%), miscarriage rate (IVF, 12.57\%; ICSI, 16.29\%), and live birth rate (IVF, 42.12\%; ICSI, 44.08\%) were similar ( $p>0.05)$. No cycles were canceled due to the formation of no usable blastocysts.

Conclusion: Although the fertilization method had no effect on clinical outcomes, the blastocyst formation rate and embryo utilization rate in the ICSI group were significantly lower than those observed in the conventional IVF group. Therefore, more care should be taken when choosing to perform blastocyst culture in ICSI patients.

Keywords: Blastocyst; In vitro fertilization; Intracytoplasmic sperm injection; Pregnancy rate

\section{Introduction}

With the development of blastocyst culture technology, elective

Received: Feb 26, 2017 · Revised: Mar 6, 2017 · Accepted: Apr 3, 2017

Co-corresponding authors: Guixue Feng

Center of Reproductive Medicine, Guangxi Maternal and Child Health Hospital, 225 Xinyang Rd, Nanning, Guangxi 530003, China

Tel: +86-13471177390 Fax:+86-07715802003 E-mail:fengguixue@126.com

\section{Bo Zhang}

Center of Reproductive Medicine, Guangxi Maternal and Child Health Hospital, 225 Xinyang Rd, Nanning, Guangxi 530003, China

Tel: +86-13978817262 Fax:+86-07715802003 E-mail: cestbon269@sina.com

*These authors contributed equally to this study.

*This work was supported by a project grant from the Natural Science Foundation of Guangxi Province (project\# 2013GXNSFAA019258), a project grant from the Research Foundation of Guangxi Medical and Health (project\# S201612) and a project grant from the Self-raised Foundation of Guangxi Health Commission (project\# Z2016093).

This is an Open Access article distributed under the terms of the Creative Commons Attribution Non-Commercial License (http://creativecommons.org/licenses/by-nc/4.0/) which permits unrestricted non-commercial use, distribution, and reproduction in any medium, provided the original work is properly cited. blastocyst culture and single blastocyst transfer have become an important way of improving the pregnancy rate and reducing the multiple pregnancy rate. However, there is no consensus regarding the inclusion criteria for blastocyst culture, especially about the effects of the insemination method on the outcome of elective blastocyst culture.

Intracytoplasmic sperm injection (ICSI), which is used mostly for severe male factor infertility and after fertilization failure in previous conventional in vitro fertilization (IVF) treatment, has been widely used. However, the ICSI procedure lacks the natural selection of sperm and bypasses the process in which spermatozoa penetrate the cumulus oophores and zona pellucida. This is unlike conventional IVF. Therefore, some concerns regarding embryo quality and pregnancy outcomes have been raised. Some research has demonstrated that the quality of embryos deriving from ICSI is inferior to the quality of those deriving from conventional IVF [1-3]. However, other studies have found that embryo quality was comparable between these insemination methods [4,5], or even superior in ICSI [6]. Therefore, it re- 
mains unclear whether ICSI affects embryo quality. It is well-known that the outcome of blastocyst culture is closely related to the quality of the embryos at the cleavage stage. Therefore, it is unclear whether with the same inclusion criteria for blastocyst culture, the culture results would be different between ICSI and conventional IVF. For this reason, the present study assessed the effects of the insemination method on the outcomes of elective blastocyst culture by retrospectively analyzing elective blastocyst culture data from January 2011 to December 2014.

\section{Methods}

\section{Experimental design and patients}

The decision to use ICSI was based on abnormal semen parameters or previous fertilization failure with conventional IVF. The inclusion criteria for elective blastocyst culture were female age $\leq 35$ years, the presence of $\geq 4$ good-quality embryos on day 3 , and $\leq 2$ retrieval cycles. Cycles with preimplantation genetic diagnosis, half-ICSI cycles, and rescue ICSI cycles were excluded. A total of 2,003 conventional IVF cycles and $336 \mathrm{ICSI}$ cycles met the inclusion criteria and underwent blastocyst culture. A retrospective analysis of these data was performed. Good-quality embryos on day 3 were defined as $\geq 6$ cells with $\leq 10 \%$ fragmentation. Blastocysts were graded according to the Gardner grading system [7]. Blastocyst grading was performed by a single trained embryologist with over 10 years of experience. Usable blastocysts were defined as having inner cell mass and trophectoderm cells with a grade of $B C / C B$ or higher. The embryo utilization rate was calculated as the number of usable blastocysts divided by the number of normally fertilized zygotes. All patients signed consent forms regarding their treatment and the study was approved by the Medical Ethics Commission of Guangxi Maternal and Child Health Hospital.

\section{Ovarian stimulation and oocyte retrieval}

Ovarian stimulation protocols were performed using the routine protocol developed by Center of Reproductive Medicine, Guangxi Maternal and Child Health Hospital. Briefly, all patients underwent downregulation with leuprolide acetate (Lupron; TAP Pharmaceuticals, Lake Forest, IL, USA). Ovarian stimulation was achieved with recombinant follicle-stimulating hormone (FSH) (Gonal-F; Merck Serono, Modugno, Italy). When two or more follicles reached a mean diameter of $18 \mathrm{~mm}$ or greater, 5,000 to $10,000 \mathrm{IU}$ of human chorionic gonadotropin (hCG) (Serono, Geneve, Switzerland or Livzon, Zhuhai, China) was administered. Oocyte retrieval was carried out 36 hours after hCG injection by vaginal ultrasound-guided puncture of the follicles.

\section{Sperm preparation}

Fresh ejaculate samples for conventional IVF were prepared by Percoll density-gradient centrifugation (ART-2024; Sage, Trumbull, CT, USA). After gradient centrifugation, the bottom fraction was aspirated and washed one time at $750 \mathrm{~g}$ for 3 minutes. After washing, the concentration and motility were assessed. Fresh ejaculate samples or epididymal/testis sperm for ICSI were prepared by centrifugation at $750 \mathrm{~g}$ for 3 minutes. The bottom fraction was then used for ICSI.

\section{Conventional IVF procedure}

For insemination, a $50-\mu \mathrm{L}$ droplet with fertilization medium and progressively motile sperm was prepared, then 4 to 6 cumulus-oocyte complexes were added into the droplet. The insemination was performed 39 to 40 hours after $\mathrm{hCG}$ injection.

\section{ICSI procedure}

Before ICSI, cumulus cells were denuded from cumulus-oocyte complexes by hyaluronidase treatment using a Pasteur pipette. The denuded oocytes were then transferred to a culture dish for ICSI. The ICSI procedure was carried out using our routine methods. ICSI was likewise performed 39 to 40 hours after hCG injection. After injection, the oocytes were transferred to culture in fertilization medium until the time of pronuclear observation.

\section{Embryo evaluation and transfer}

Fertilization was usually confirmed 16 to 18 hours postinsemination (day 1). Cumulus cells were denuded from conventional IVF-inseminated oocytes using a Pasteur pipette. In both conventional IVF and ICSI, only zygotes with two pronuclei were transferred to an individual droplet of Quinn's cleavage medium (ART-1026, Sage), Quinn's blastocyst culture medium (ART-1029, Sage) for further culture. On day 3 , the cleavage embryos were scored. According to the patient's condition and the cleavage ebryo quality, after informed consent, patients with a good prognosis were advised to delay the culture to the blastocyst stage. All cleavage embryos were then moved to Quinn's blastocyst culture media (Sage) for further culture until day 5 or day 6 . If the patient was in a suitable condition for transplantation, intrauterine embryo replacement was carried out on day 5 or day 6 with the best blastocyst. Otherwise, all usable blastocysts were cryopreserved for frozen cycles. All embryos were cultured in low oxygen with $5 \% \mathrm{O}_{2}$.

\section{Statistical analysis}

All analyses were performed with the use of SPSS ver. 13.0 (SPSS Inc., Chicago, IL, USA). Statistical analysis was performed with the Student $t$-test and the chi-square test. Significance was set at $p<$ 0.05 . 
Table 1. Demographics and outcomes of blastocyst culture in the IVF and ICSI groups

\begin{tabular}{lccc}
\hline Variable & IVF & ICSI & $p$-value \\
\hline No. of cycles & 2,003 & 336 & - \\
Mean age $(\mathrm{yr})$ & $29.75 \pm 3.13$ & $29.41 \pm 3.36$ & 0.081 \\
Basal FSH $(\mathrm{IU} / \mathrm{mL})$ & $6.85 \pm 1.73$ & $6.73 \pm 1.46$ & 0.236 \\
Basal LH $(\mathrm{IU} / \mathrm{mL})$ & $6.08 \pm 3.75$ & $6.36 \pm 3.75$ & 0.245 \\
Average BMI $\left(\mathrm{kg} / \mathrm{m}^{2}\right)$ & $21.02 \pm 2.59$ & $20.98 \pm 2.59$ & 0.808 \\
No. of oocytes & $17.73 \pm 7.16$ & $17.69 \pm 6.92$ & 0.923 \\
Maturation rate & $29,054 / 35,521(81.79)$ & $4,770 / 5,845(81.61)$ & 0.733 \\
Fertilization rate & $25,652 / 35,521(72.22)$ & $4,164 / 5,845(71.24)$ & 0.123 \\
Good-quality embryo rate & $15,280 / 25,652(59.57)$ & $2,430 / 4,164(58.36)$ & 0.141 \\
Blastocyst formation rate & $14,032 / 25,652(54.70)$ & $2,121 / 4,164(50.94)$ & 0.001 \\
Embryo utilization rate & $13,106 / 25,652(51.09)$ & $1,984 / 4,164(47.65)$ & 0.001 \\
\hline
\end{tabular}

Values are presented as mean \pm standard deviation or number (\%).

IVF, in vitro fertilization; ICSI, intracytoplasmic sperm injection; FSH, follicle-stimulating hormone; LH, luteinizing hormone; BMI, body mass index.

Table 2. Comparison of clinical outcomes between the IVF and ICSI groups

\begin{tabular}{lccc}
\hline Variable & IVF & ICSI & $p$-value \\
\hline No. of embryo-transfer cycles & 1,453 & 245 & - \\
Endometrial thickness $(\mathrm{mm})$ & $11.46 \pm 2.25$ & $11.70 \pm 2.22$ & 0.119 \\
Embryos replaced per cycle & $1.00 \pm 0.00$ & $1.00 \pm 0.00$ & - \\
Clinical pregnancy rate & $740 / 1,453(50.93)$ & $135 / 245(55.10)$ & 0.24 \\
Miscarriage rate & $93 / 740(12.57)$ & $22 / 135(16.29)$ & 0.267 \\
Ectopic pregnancy rate & $31 / 740(4.19)$ & $5 / 135(3.70)$ & 0.794 \\
Live birth rate & $612 / 1,453(42.12)$ & $108 / 245(44.08)$ & 0.577 \\
\hline
\end{tabular}

Values are presented as mean \pm standard deviation or number (\%).

IVF, in vitro fertilization; ICSI, intracytoplasmic sperm injection.

\section{Results}

A total of 2,003 conventional IVF and 336 ICSI cycles were included in this study. Table 1 summarizes the outcomes of our study. The mean age of female patients, the basal FSH level, the basal luteinizing hormone level, and average body mass index were similar between these groups.

In the conventional IVF group, 25,652 of the 35,521 oocytes were fertilized. The mean fertilization rate among retrieved oocytes was $72.22 \%$. In the ICSI group, 4,770 of the 5,845 oocytes were metaphase II and were injected with sperm; of these, 4,164 oocytes were fertilized. On the basis of the number of oocytes, there was no significant difference in the fertilization rate between the conventional IVF and ICSI groups ( $72.22 \%$ vs. $71.24 \%, p>0.05)$. Overall, 25,652 embryos in the conventional IVF group and 4,164 embryos in the ICSI group progressed to blastocysts, and the good-embryo rate on day 3 was similar (IVF, 59.57\%; ICSI, 58.36\%, p>0.05), but the blastocyst formation rate was significantly different. In the conventional IVF group, 14,032 blastocysts were formed, including 13,106 usable blastocysts; in contrast, there were 2,121 blastocysts, including 1,984 usable blastocysts, in the ICSI group. Therefore, the blastocyst formation rate among fertilized oocytes in the conventional IVF group was significantly higher than in the ICSI group $(54.70 \%$ vs. $50.94 \%, p<0.05)$ and the embryo utilization rate was also better in the conventional IVF group $(51.09 \%$ vs. $47.65 \%, p<0.05)$.

Regarding clinical parameters, no differences were found in the clinical pregnancy rate (IVF, 50.93\%; ICSI, 55.10\%), the miscarriage rate (IVF, 12.57\%; ICSI, 16.29\%), the ectopic pregnancy rate (IVF, 4.19\%; ICSI, $3.70 \%)$, or the live birth rate (IVF, 42.12\%; ICSI, 44.08\%) ( $p>0.05)$ (Table 2).

\section{Discussion}

As is known, the ultimate goal of assisted reproductive technology is for the patient to take one term healthy baby home per cycle [8]. Therefore, single-embryo transfer was proposed. Furthermore, elective blastocyst culture and single blastocyst transfer have been demonstrated as the best way to achieve this goal [9-11]. However, blastocyst culture is not suitable for all patients, so choosing patients appropriately is critical. Nonetheless, there are no uniform inclusion criteria for blastocyst culture. The parameters of the inclusion criteria have included the number of oocytes retrieved, the number of good 
embryos on day 3, and the patients' age [12]. No direct analysis has been conducted on the effect of the insemination method.

Therefore, the main purpose of this study was to compare the effect of different fertilization methods on the outcomes of elective blastocyst culture. No significant differences were found between conventional IVF and ICSI cycles in the mean age, basal levels of FSH or luteinizing hormone, body mass index, the number of oocytes, or the fertilization rate. All included cycles progressed to the blastocyst stage, and the good-embryo rate on day 3 was similar. Therefore, the main influencing factor in this study was the insemination method. Our data demonstrated that blastocyst formation in vitro was affected by the insemination method. The blastocyst formation rate in the ICSI group was significantly lower than in the conventional IVF group. This finding also confirmed the results that have been reported by others [1,3,13]. Griffiths et al. [3] used sibling oocytes with the same semen sample for conventional IVF or ICSI, and demonstrated that the ICSI procedure contributed to a reduced capacity for blastocyst formation. Other studies using surplus embryos $[1,13,14]$, which were not able to control for potential confounders such as surplus embryo quality, also showed that blastocyst formation was compromised by ICSI. However, some other studies did not support the conclusion that ICSI decreased the blastocyst formation rate $[5,15]$. This study, to our knowledge, was the first to only include cycles in which elective blastocyst culture was performed.

The different blastocyst formation rate in conventional IVF and ICSI is mainly related to the different binding mechanism of the egg and sperm. During conventional IVF fertilization, natural selection of sperm takes place, just like in natural fertilization, in which the sperm must penetrate the oocyte-corona-cumulus complex, zona pelluci$\mathrm{da}$, and plasmalemma, and only then can the sperm and oocyte be combined. However, in the ICSI procedure, the operation bypasses such selection, and so-called 'forced fertilization' can be achieved using spermatozoa that might never have attained natural fertilization or with genetic defects. In theory, embryonic development is influenced by the quality of the sperm and egg. The first three cleavage divisions are mainly regulated by maternal genes, and then at the 4to 8-cell stage of development, the embryonic genome is activated and the paternal genome starts to play a role [16]. Many studies have demonstrated that spermatozoa from patients with severe male infertility had an adverse effect on blastocyst formation [17]. This may be related to the fact that spermatozoa from infertile men have higher rates of defects such as DNA damage and aneuploidy. Fluorescent in situ hybridization results have shown that chromosomal aneuploidy was related to the presence of sperm with abnormal characteristics, such as round-headed sperm and large-headed sperm [18]. Therefore, the embryo quality on day 3 may be associated with oocyte-related factors, while genetically abnormal sperm may show a negative influence on blastulation. Our study also demonstrated that the good-embryo rates on day 3 in the conventional IVF and ICSI groups were similar, while the blastocyst formation rate in the ICSI group was significantly worse. This may have occurred because the condition of the female patients included in these groups was similar, but the semen parameters in the ICSI group were poorer. Inconsistent conclusions have been made about the effect of sperm on early embryo development. Some researchers have shown that severe teratozoospermia had no effect on blastocyst formation $[19,20]$. In addition, the ICSI operation itself may be detrimental to the oocyte, and furthermore to the development of the embryo. A study by Dumoulin et al. [21] indicated that the volume of aspirated cytoplasm, type of membrane breakage, and skill in performing ICSI had an effect on blastocyst formation. Meanwhile, manipulation of the degranulated cell and the injection of polyvinylpyrrolidone and culture medium into the oocyte may also influence the subsequent development of the embryo. Furthermore, the injection needle may injure the spindle, which may cause errors in the first cleavage division, then lead to aneuploidy. A paper showed that ICSI affected embryonic development and gene expression in cattle [22]. However, another animal model reported that ICSI did not perturb embryonic development [23].

Our study compared the clinical outcomes between conventional IVF and ICSI groups, and showed that there were no differences in the clinical pregnancy/implantation rate, miscarriage rate, or live birth rate. These findings suggest that the subsequent developmental potential of blastocysts derived from ICSI did not appear to be affected. These results are also supported by those of other studies $[14,24]$. It may be beneficial to delay culture to day 5 or 6 , such that some chromosomally abnormal embryos fail to undergo blastulation in culture.

In conclusion, blastocyst formation ability was significantly affected by ICSI. Therefore, in order to avoid cases of cycle cancellation due to the formation of no blastocysts, it can be suggested that more care should be taken in choosing blastocyst culture for ICSI patients.

\section{Conflict of interest}

No potential conflict of interest relevant to this article was reported.

\section{Acknowledgments}

We thank the clinicians, clinical embryologists, laboratory technologists, and nurses of the Centre of Reproductive Medicine for data collection. 


\section{References}

1. Miller JE, Smith TT. The effect of intracytoplasmic sperm injection and semen parameters on blastocyst development in vitro. Hum Reprod 2001;16:918-24.

2. Hsu Ml, Mayer J, Aronshon M, Lanzendorf S, Muasher S, Kolm P, et al. Embryo implantation in in vitro fertilization and intracytoplasmic sperm injection: impact of cleavage status, morphology grade, and number of embryos transferred. Fertil Steril 1999;72: 679-85.

3. Griffiths TA, Murdoch AP, Herbert M. Embryonic development in vitro is compromised by the ICSI procedure. Hum Reprod 2000; 15:1592-6.

4. Yoeli R, Orvieto R, Ashkenazi J, Shelef M, Ben-Rafael Z, Bar-Hava I. Comparison of embryo quality between intracytoplasmic sperm injection and in vitro fertilization in sibling oocytes. J Assist Reprod Genet 2008;25:23-8.

5. Van Landuyt L, De Vos A, Joris H, Verheyen G, Devroey P, Van Steirteghem A. Blastocyst formation in in vitro fertilization versus intracytoplasmic sperm injection cycles: influence of the fertilization procedure. Fertil Steril 2005;83:1397-403.

6. Khamsi F, Yavas Y, Roberge S, Wong JC, Lacanna IC, Endman M. Intracytoplasmic sperm injection increased fertilization and good-quality embryo formation in patients with non-male factor indications for in vitro fertilization: a prospective randomized study. Fertil Steril 2001;75:342-7.

7. Gardner DK, Lane M, Stevens J, Schlenker T, Schoolcraft WB. Blastocyst score affects implantation and pregnancy outcome: towards a single blastocyst transfer. Fertil Steril 2000;73:1155-8.

8. Min JK, Breheny SA, MacLachlan V, Healy DL. What is the most relevant standard of success in assisted reproduction? The singleton, term gestation, live birth rate per cycle initiated: the BESST endpoint for assisted reproduction. Hum Reprod 2004;19: 3-7.

9. Teranishi A, Kuwata A, Fumino T, Hamai H, Shigeta M. A theoretical model for single blastocyst transfer. J Assist Reprod Genet 2009;26:327-34.

10. Sills ES, Palermo GD. Human blastocyst culture in IVF: current laboratory applications in reproductive medicine practice. Rom J Morphol Embryol 2010;51:441-5.

11. Styer AK, Wright DL, Wolkovich AM, Veiga C, Toth TL. Single-blastocyst transfer decreases twin gestation without affecting pregnancy outcome. Fertil Steril 2008;89:1702-8.

12. Thum MY, Wells V, Abdalla H. Patient selection criteria for blastocyst culture in IVF/ICSI treatment. J Assist Reprod Genet 2010;
27:555-60.

13. Dumoulin JC, Coonen E, Bras M, van Wissen LC, Ignoul-Vanvuchelen R, Bergers-Jansen JM, et al. Comparison of in-vitro development of embryos originating from either conventional invitro fertilization or intracytoplasmic sperm injection. Hum Reprod 2000;15:402-9.

14. Shoukir Y, Chardonnens D, Campana A, Sakkas D. Blastocyst development from supernumerary embryos after intracytoplasmic sperm injection: a paternal influence? Hum Reprod 1998;13: 1632-7.

15. Westphal LM, Hinckley MD, Behr B, Milki AA. Effect of ICSI on subsequent blastocyst development and pregnancy rates. J Assist Reprod Genet 2003;20:113-6.

16. Tesarik J. Paternal effects on cell division in the human preimplantation embryo. Reprod Biomed Online 2005;10:370-5.

17. Balaban B, Urman B, Isiklar A, Alatas C, Mercan R, Aksoy S, et al. Blastocyst transfer following intracytoplasmic injection of ejaculated, epididymal or testicular spermatozoa. Hum Reprod 2001; 16:125-9.

18. Miharu N. Chromosome abnormalities in sperm from infertile men with normal somatic karyotypes: oligozoospermia. Cytogenet Genome Res 2005;111:347-51.

19. French DB, Sabanegh ES Jr, Goldfarb J, Desai N. Does severe teratozoospermia affect blastocyst formation, live birth rate, and other clinical outcome parameters in ICSI cycles? Fertil Steril 2010;93:1097-103.

20. Berger DS, Abdelhafez F, Russell H, Goldfarb J, Desai N. Severe teratozoospermia and its influence on pronuclear morphology, embryonic cleavage and compaction. Reprod Biol Endocrinol 2011;9:37.

21. Dumoulin JM, Coonen E, Bras M, Bergers-Janssen JM, IgnoulVanvuchelen RC, van Wissen LC, et al. Embryo development and chromosomal anomalies after ICSI: effect of the injection procedure. Hum Reprod 2001;16:306-12.

22. Arias ME, Risopatron J, Sanchez R, Felmer R. Intracytoplasmic sperm injection affects embryo developmental potential and gene expression in cattle. Reprod Biol 2015;15:34-41.

23. Piotrowska-Nitsche K, Chan AW. Effect of sperm entry on blastocyst development after in vitro fertilization and intracytoplasmic sperm injection: mouse model. J Assist Reprod Genet 2013;30: 81-9.

24. Karaki RZ, Samarraie SS, Younis NA, Lahloub TM, Ibrahim MH. Blastocyst culture and transfer: a step toward improved in vitro fertilization outcome. Fertil Steril 2002;77:114-8. 